\title{
O efeito dos exopolissacarídeos obtidos a partir de bactérias ácido lácticas como prebiótico: uma revisão sistemática
}

\author{
The effect of exopolysaccharides obtained from lactic acid bacteria as a prebiotic: a systematic
} review

\author{
El efecto de los exopolisacáridos obtenidos a partir de bacterias del ácido láctico como prebiótico: \\ una revisión sistemática
}

Rejane Gonçalves Monteiro ORCID: https://orcid.org/0000-0002-1716-5675 Universidade Federal Rural de Pernambuco, Brasil E-mail: rejane_bio@hotmail.com Elaine Cristina da Silva ORCID: https://orcid.org/0000-0003-2395-7166 Universidade Federal Rural de Pernambuco, Brasil E-mail: e.csilva2@outlook.com

Anna Larissa Cerqueira Martins ORCID: https://orcid.org/0000-0003-0141-1314 Universidade Federal Rural de Pernambuco, Brasil E-mail: annalarissa01@gmail.com

Ana Lúcia Figueiredo Porto ORCID: https://orcid.org/0000-0001-5561-5158 Universidade Federal Rural de Pernambuco, Brasil E-mail: analuporto@yahoo.com

Maria Taciana Cavalcanti Vieira Soares

ORCID: https://orcid.org/0000-0001-9573-6296 Universidade Federal Rural de Pernambuco, Brasil E-mail:mtcvsoares@yahoo.com

\begin{abstract}
Resumo
Exopolissacarídeos de bactérias ácido láticas são biopolímeros conhecidos pela diversidade estrutural e devido a isto eles apresentam vários efeitos benéficos a saúde humana, podendo atuar como imunomoduladores, antioxidantes, antitumorais etc. Sabe-se que os exopolissacarídeos também auxiliam no equilíbrio da microbiota intestinal, porém ainda não há constatação de que seja por efeito prebiótico. Sendo assim, este estudo teve por objetivo avaliar o desempenho dos exopolissacarídeos produzidos por Bactérias ácido láticas como prebióticos por meio de uma revisão sistemática de literatura. A revisão foi realizada adotando a estratégia PRISMA. Foram coletados estudos realizados entre 2011 e 2020, utilizando 4 diferentes bases de dados. As palavras chaves empregadas foram: "exopolysaccharides", "prebiotics", "prebiotic potential", "prebiotic effects", "lactic acid bacteria" e "probiotics". Foram analisadas ao todo, 7 referências relativas aos efeitos prebióticos de EPS em geral, e destes poucos realizaram estudos clínicos randomizados e controlados. Embora os EPS estudados tenham apresentado características típicas de um prebiótico, ainda há a necessidade de estudos futuros com melhor delineamento experimental para verificar com maior precisão esses efeitos, bem como a determinação da duração e dosagens adequadas.
\end{abstract}

Palavras-chave: Bactéria ácido lática; Exopolissacarídeo; Prebiótico.

\begin{abstract}
Exopolysaccharides from lactic acid bacteria are biopolymers known for their structural diversity and because of this they have several beneficial effects on human health, and can act as immunomodulators, antioxidants, antitumors etc. It is known that exopolysaccharides also help in the balance of the intestinal microbiota, but there is still no evidence that it is due to a prebiotic effect. Therefore, this study aimed to evaluate the performance of exopolysaccharides produced by lactic acid bacteria as prebiotics through a systematic literature review. The review was carried out adopting the PRISMA strategy. Studies carried out between 2011 and 2020 were collected, using 4 different databases. The key words used were: "exopolysaccharides", "prebiotics", "prebiotic potential", "prebiotic effects", "lactic acid bacteria" and "probiotics". A total of 7 references were analyzed regarding the prebiotic effects of EPS in general, and of these few, randomized and controlled clinical studies were carried out. Although the EPS studied have presented typical characteristics of a prebiotic, there is still a need for future studies with a better experimental design to verify these effects with greater precision, as well as to determine the appropriate duration and dosages.
\end{abstract}

Keywords: Lactic acid bactéria; Exopolysaccharide; Prebiotic. 


\begin{abstract}
Resumen
Los exopolisacáridos de las bacterias del ácido láctico son biopolímeros conocidos por su diversidad estructural y por ello tienen varios efectos beneficiosos sobre la salud humana, pudiendo actuar como inmunomoduladores, antioxidantes, antitumorales, etc. Se sabe que los exopolisacáridos también ayudan en el equilibrio de la microbiota intestinal, pero aún no hay evidencia de que se deba a un efecto prebiótico. Por lo tanto, este estudio tuvo como objetivo evaluar el desempeño de los exopolisacáridos producidos por las bacterias del ácido láctico como prebióticos a través de una revisión sistemática de la literatura. La revisión se realizó adoptando la estrategia PRISMA. Se recogieron estudios realizados entre 2011 y 2020, utilizando 4 bases de datos diferentes. Las palabras clave utilizadas fueron: "exopolisacáridos", "prebióticos", "potencial prebiótico", "efectos prebióticos", "bacterias del ácido láctico" y "probióticos". Se analizaron un total de 7 referencias sobre los efectos prebióticos de los SEP en general, y de estos pocos se realizaron estudios clínicos aleatorizados y controlados. Si bien los EPS estudiados han presentado características típicas de un prebiótico, aún se necesitan estudios futuros con un mejor diseño experimental para verificar estos efectos con mayor precisión, así como para determinar la duración y dosis adecuadas.
\end{abstract}

Palabras clave: Bacterias del ácido láctico; Exopolisacárido; Prebiótico.

\title{
1. Introdução
}

Os exopolissacarídeos (EPS) produzidos por Bactérias Ácido Láticas (BAL) possuem características físico-químicas, através das quais proporcionam melhorias nas texturas e nas propriedades organolépticas melhorando a qualidade do produto fabricado nas indústrias alimentícias, de cosméticos, entre outras (Lynch, et al., 2018).

Para a indústria, os EPS são de grande interesse comercial, pois ajudam na purificação ao final dos bioprocessos, possuem propriedades físico-químicas reprodutíveis, além de existirem em grande abundância (Jindal, Singh \& Khattan, 2018). Também são considerados essenciais fontes de hidrocolóides (gomas alimentares) por proporcionar estrutura e estabilidade quando aplicados na produção de alimentos (Gao, et al., 2017).

Existem muitas aplicações industriais potenciais para EPS originado por BAL, como seu papel como gelificante, espessantes, emulsificantes, estabilizantes, aglutinantes de água e agentes viscosificantes. Além disso, o EPS produzido pela BAL tem um papel potencial na preparação de produtos lácteos e à base de cereais (Daba, Elnahas \& Elkhateeb, 2021).

No âmbito de reologia, os EPS influenciam na capacidade de biopolímero, pois dão maior uniformidade na estrutura química do composto, considerando o tamanho e a frequência das ramificações e a massa molecular (Zhou, et al., 2018). Podem ter ação prebiótica, propriedade esta, que ainda se encontra em estudo para garantir sua eficácia (Caggianiello, et al., 2016).

A ação prebiótica diz respeito ao crescimento de forma seletiva de microrganismos benéficos da microbiota intestinal assim como de probióticos. A modulação dessa microbiota, e a inclusão de probióticos na dieta, podem ser aproveitadas em terapias alternativas para a prevenção e tratamento de doenças (Arora, Green \& Prakash, 2020; Carmo, 2019) e vêm se tornando cada vez mais comum nos suplementos alimentícios para favorecer a saúde em geral dos seres humanos (Renschler, et al., 2020).

Assim, o objetivo dessa revisão sistemática foi investigar o uso de exopolissacarídeos produzidos a partir das bactérias ácido láticas como prebióticos.

\section{Metodologia}

\subsection{Diretrizes}

Esta revisão sistemática seguiu às diretrizes determinadas pelo Preferred Reporting Items for Systematic Reviews and Meta-Analyses (PRISMA). (Moher, et al., 2009).

\subsection{Critérios de elegibilidade}

Os artigos científicos selecionados para esta revisão sistemática foram aqueles que desenvolviam estudos primários, publicados em língua inglesa nos últimos 10 anos, 2011 a 2020, que associaram a administração de exopolissacarídeo produzidos por Bals com ação prebiótica em ensaios in vitro. Foram excluídos da revisão sistemática artigos de estudo secundário (revisões, relatórios e livros) e duplicados, assim como estudos que não possuíam todos os critérios de elegibilidade, tais como: ensaios in 
vivo, que não utilizaram o exopolissacarídeo como prebiótico em teste.

\subsection{Estratégias de pesquisa e identificação do estudo}

A seleção dos artigos foi realizada por três revisores que o fizeram de forma independente, tomando como base três etapas: título, seguida de resumo e por fim o texto completo, a fim de verificar os critérios de elegibilidade. Na pesquisa de dados as seguintes palavras-chave foram utilizadas: "exopolysaccharides", "prebiotics", "prebiotic potential", "prebiotic effects", "lactic acid bacteria" e "probiotics". Para obter maior alcance dos resultados, os termos foram interligados com a inserção dos operadores "or" e "and". Devido a heterogeneidade dos estudos selecionados e qualidade do ensaio, não foi feita uma metaanálise, logo os resultados foram resumidos em texto.

\section{Resultados e Discussão}

\subsection{Artigos pesquisados}

Na Figura 1 encontra-se um fluxograma para melhor entendimento do processo de seleção dos trabalhos. Onde, podemos observar que dos 487 artigos, inicialmente obtidos nas bases de dados Pubmed/ Medline/Web of science/ Scopus /Science Direct, a partir da utilização dos critérios de seleção e elegibilidade, restaram 7 artigos que passaram por uma leitura cuidadosa.

Figura 1: Fluxograma de identificação e seleção dos artigos para revisão sistemática sobre o efeito prebiótico de exopolissacarídeos, 2011-2020.

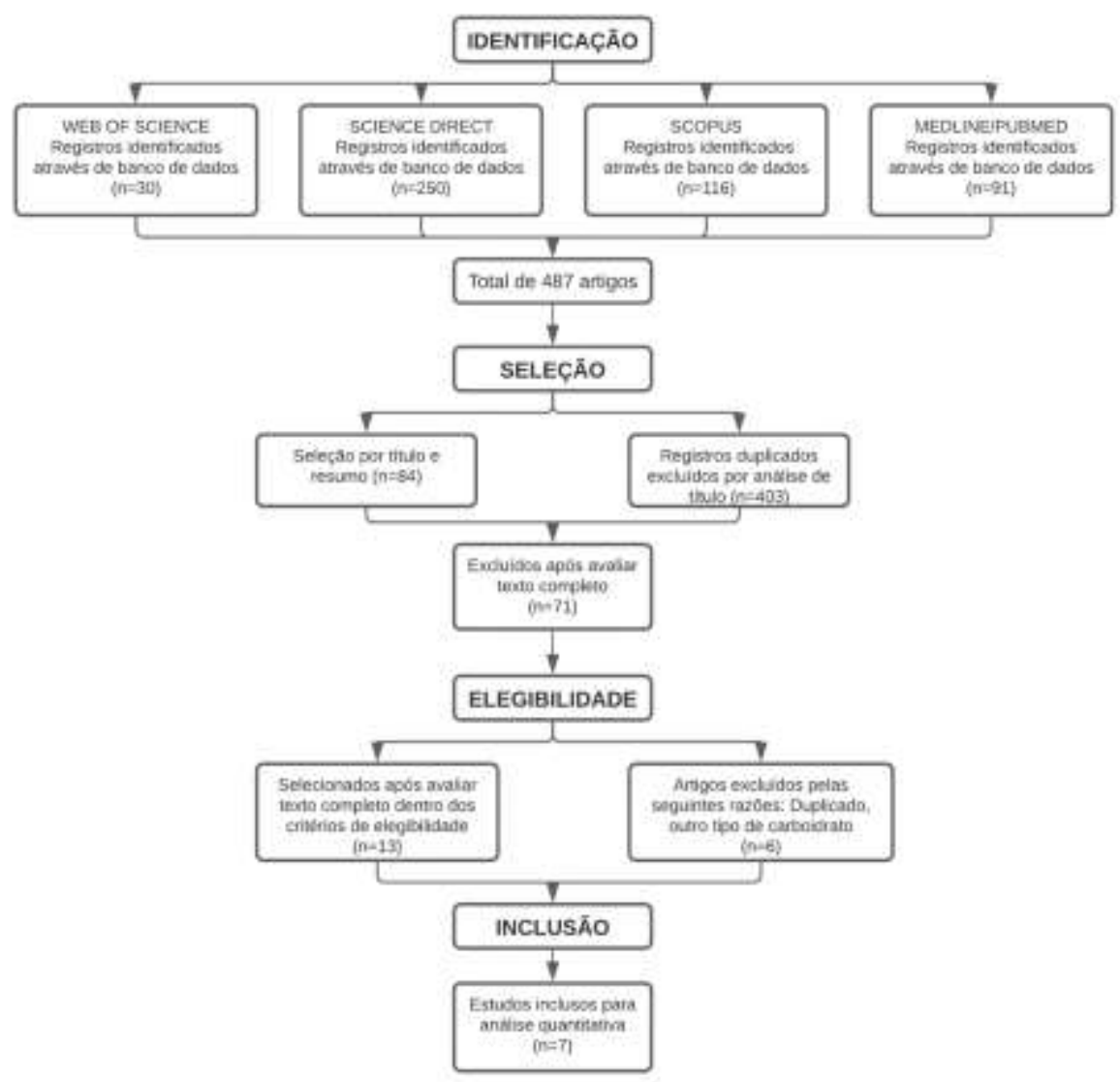

Fonte: Autores (2020).

Este fluxograma traz informações de como se deu a pesquisa da revisão sistemática de forma sucinta seguindo os critérios para a seleção dos estudos apresentados usando os protocolos para a realização da revisão sistemática. 
Os artigos incluídos analisaram a influência dos exopolissacarídeos com ação prebiótica na microbiota intestinal em ensaios in vitro. Na Tabela 1 tem-se um compilado de informações relevantes sobre os artigos analisados e os pontos principais para análise desse efeito prebiótico do EPS.

Tabela 1: Dados avaliados nos artigos utilizados neste estudo.

\begin{tabular}{|c|c|c|c|c|c|c|c|}
\hline Referências & $\begin{array}{l}\text { Microrganismos } \\
\text { produtores de } \\
\text { EPS }\end{array}$ & EPS & $\begin{array}{c}\text { Carboidrato } \\
\text { padrão para } \\
\text { crescimento dos } \\
\text { probióticos } \\
\end{array}$ & $\begin{array}{l}\text { Concentraçã } \\
\text { o do EPS } \\
\text { como } \\
\text { prebiótico } \\
\end{array}$ & Produção de ácido graxo & $\begin{array}{c}\text { Tempo de } \\
\text { crescimento dos } \\
\text { microrganismos } \\
\text { probióticos } \\
\end{array}$ & Resultados (Efeito Prebiótico) \\
\hline $\begin{array}{l}\text { Liu } \text { et al., } \\
\text { (2020) }\end{array}$ & Erwinia sp. 10119 & Levana & Inulina & NR & $\begin{array}{l}\text { Aumento do acetato e do butirato, } \\
\text { respectivamente, de } 14.16 \mathrm{mM} \text { para } \\
30.37 \mathrm{mM} \text { e de } 26.03 \mathrm{mM} \text { para } \\
65.41 \mathrm{mM} \text {. }\end{array}$ & $48 \mathrm{~h}$ & $\begin{array}{l}\text { A levana influenciou no crescimento da } \\
\text { população de Bifidobacterium, mas não } \\
\text { houve mudança significativa na população de } \\
\text { Lactobacillus. }\end{array}$ \\
\hline $\begin{array}{l}\text { Tang et al., } \\
\quad\left(2020^{1}\right)\end{array}$ & $\begin{array}{c}\text { Lactobacillus } \\
\text { delbrueckii ssp.e } \\
\text { Lactobacillus } \\
\text { bulgaricus. } \\
\end{array}$ & NR & Inulina & $1 \%$ & $\begin{array}{l}\text { Ao final das } 48 \mathrm{~h} \text { de fermentação, o } \\
\text { total de AGCC de r-eps } 1 \text {, r-eps } 2 \mathrm{e} \\
\text { inulina foram, respectivamente, } \\
145 \mathrm{mM}, 135 \mathrm{mM} \text { e } 130 \mathrm{mM} \text {. }\end{array}$ & $48 \mathrm{~h}$ & $\begin{array}{l}\text { Os EPSs testados apresentaram forte efeito } \\
\text { prebiótico em comparação ao padrão. }\end{array}$ \\
\hline $\begin{array}{l}\text { Tang et al., } \\
\quad\left(2020^{2}\right)\end{array}$ & $\begin{array}{l}\text { Lactobacillus } \\
\text { delbrueckii ssp. } \\
\text { bulgaricus }\end{array}$ & NR & Inulina e Glicose & NR & NR & $48 \mathrm{~h}$ & $\begin{array}{l}\text { Não foi observado crescimento de } \\
\text { Bifidobacterium. }\end{array}$ \\
\hline $\begin{array}{l}\text { Hussein et al., } \\
\quad \text { (2015) }\end{array}$ & $\begin{array}{c}\text { Lactobacillus } \\
\text { delbrueckii } \\
\text { bulgaricus, } \\
\text { Lactobacillus } \\
\text { helveticus e } \\
\text { Lactobacillus casei }\end{array}$ & NR & NR & NR & NR & $\begin{array}{l}\text { 24h, 48h, 72h, } \\
96 \mathrm{~h}, 120 \mathrm{~h}\end{array}$ & $\begin{array}{l}\text { Maior efeito prebiótico com o EPS de } \\
\text { Lactobacillus delbrueckii bulgaricus. }\end{array}$ \\
\hline $\begin{array}{l}\text { Das et al., } \\
\quad(2014)\end{array}$ & $\begin{array}{l}\text { Lactobacillus } \\
\text { plantarum DM5 }\end{array}$ & Glucano & Inulina & $1 \%$ & NR & $24 \mathrm{~h}$ & $\begin{array}{l}\text { Aumento significativo das populações de } \\
\text { Bifidobacterium Infantis e Lactobacillus. } \\
\text { sp. }\end{array}$ \\
\hline $\begin{array}{l}\text { Grosu-Tudor et } \\
\text { al., (2013) }\end{array}$ & $\begin{array}{l}\text { Lactococcus lactis, } \\
\text { Leuconostoc } \\
\text { citreum, Weissella } \\
\text { confusa/cibaria, } \\
\text { Streptococcus } \\
\text { thermophilus } \\
\end{array}$ & NR & Lactose & $5 \mathrm{~g} / \mathrm{L}$ & $\begin{array}{l}\text { A produção de acetato foi de } 12 \\
\mathrm{mM} / \mathrm{L} .\end{array}$ & $92 \mathrm{~h}$ & $\begin{array}{l}\text { Apenas os HoPs mostraram efeito } \\
\text { bifidogênico. }\end{array}$ \\
\hline $\begin{array}{l}\text { Hongpattaraker } \\
\text { e et al., (2012) }\end{array}$ & $\begin{array}{l}\text { Weissella cibaria, } \\
\text { Weissella } \\
\text { confusa,Lactobacil } \\
\text { lus plantarume } \\
\text { Pediococcus } \\
\text { pentosaceus }\end{array}$ & NR & Glicose & $1 \%$ & $\begin{array}{c}\text { Após } 24 \mathrm{~h} \text { de simulação, a } \\
\text { produção de ácido acético foi de } \\
10 \mathrm{~L} \text {. }\end{array}$ & $24 \mathrm{~h}, 48 \mathrm{~h}$ & $\begin{array}{l}\text { O EPS de Weissella cibaria foi o substrato } \\
\text { que estimulou o crescimento de Bifidum } \\
\text { bifido, de } 6.17 \text { para } 7.54 \log C F U / m L \text {. }\end{array}$ \\
\hline
\end{tabular}

NR: Não Referenciado. Fonte: Autores (2021). 
Nesta tabela observa-se os resultados dos artigos analisados com suas respectivas informações relevantes para o estudo, como a produção de EPS e tempo de cultivo do microorganismo, produção de ácido graxos, fonte de carbono e efeito prebiótico.

\subsection{Efeito prebiótico}

A maioria dos estudos demonstram que o exopolissacarídeo tem efeito prebiótico devido ao seu efeito bifidogênico e ao estímulo do crescimento das bactérias ácido láticas, como Lactobacillus spp. (Hongpattarakere, et al., 2012; Das, et al., 2014; Tang, et al., 20201).

De acordo com estudos avaliados foi observado a falta ou baixo estímulo do crescimento de bactérias patogênicas como Clostridium spp., Escherichia coli e Bacteroides com as suplementações de exopolissacarídeos aos meios de cultura. O não crescimento desses microrganismos sugere que o prebiótico serve como fonte de carbono apenas para probióticos, atingindo assim sua funcionalidade final (Hongpattarakere, et al., 2012; Grosu- Tudor et al., 2013; Das, et al., 2014; Liu, et al., 2020; Tang, et al., 2020²).

Foi identificado que os exopolissacarídeos que serviram como melhores fontes de carbono para bactérias probióticas foram os homopolissacarídeos, devido o mesmo ser resistente a passagem gastrointestinal e não sofrer degradações significativas, podendo-se dizer que possuem efeito de fibra (Grosu-Tudor, et al., 2013 e Tang, et al., 20201). Entretanto, foi observado que heteropolissacarídeos formados por glicose e arabinose, produzidos por Lactobacillus delbrueckii spp. bulgaricus, apresentam alto índice como prebiótico (Hussein, et al., 2015).

O estudo de Das, et al. (2014) é pioneiro na análise da ação prebiótica do glucano DM5 produzido a partir de $L$. plantarum DM5. O glucano DM5 em comparação com a goma de guar, que já é muito utilizada na indústria, se mostra como bom biofloculante e emulsificante, por suportar altas temperaturas, ter uma boa retenção de água, e apresentar uma estrutura formada por polissacarídeos. Ele também estimula o crescimento de L. plantarum DM5, L. acidophilus NRRL B-4496 e B. infantis NRRL B-41661, em comparação com a inulina, que já é considerado prebiótico.

Foi analisado a taxa de crescimento de Bifidobacterium a partir de EPS produzidos por Lactobacillus delbrueckii ssp. bulgaricus SRFM-1 (Tang, et al., 20201; Hussein, et al., 2015), Lactococcus lactis (Grosu-Tudor, et al., 2013), Weissella cibaria A2 (Hongpattarakere, et al., 2012) e Erwinia spp. 10119 (Liu, et al., 2020) e em todos os casos houve aumento populacional significativo, o que demonstra uma eficácia da ação prebiótica dos EPS produzidos por essas cepas.

Estudos que analisaram frações de exopolissacarídeos produzidas por Lactobacillus delbrueckii ssp. bulgaricus SRFM1 mostram sua eficiência como fontes de carbono para o crescimento de bactérias do gênero Lactobacillus, como a L. plantarum 70810 e a L. casei ssp. rhamnosus LS-8. Assim como conseguem reduzir o volume de células de Escherichia coli e não estimulam o crescimento de Streptococcus thermophilus (Tang, et al., 20202).

\subsection{Produção de ácidos graxos de cadeia curta (AGCC)}

Os ácidos graxos de cadeia curta são essenciais para a saúde do hospedeiro, e por isso a avaliação de sua produção oriunda da fermentação de prebióticos se torna um fator importante na análise do potencial destes suplementos. Alguns exemplos da atuação dos AGCC no organismo são: o ácido butírico, que previne contra o câncer colo retal e é fonte de energia para a microbiota intestinal, o ácido propiônico que reduz a síntese de colesterol no fígado e aumenta o metabolismos de lipídeos e o ácido acético que é oxidado pelo coração (Maldonado-Contreras, et al., 2020; Gibson, et al., 2017).

A concentração de ácidos graxos de cadeia curta, principalmente de ácido acético, foi um parâmetro avaliado nos resultados da suplementação de exopolissacarídeo como prebiótico, pois, esses são produzidos a partir da fermentação de fontes de carbono pela microbiota intestinal e, como supracitado, tem um grande fator de importância para a saúde do hospedeiro (Hongpattarakere, et al., 2012; Grosu- Tudor, et al., 2013; Liu, et al., 2020; Tang, et al., 20201). 
Grosu-Tudor, et al. (2013) relacionou a baixa produção de ácido acético, 12mM/litro, ao baixo crescimento de Bifidobacterium spp. o qual foi suplementado com exopolissacarídeo produzido por Lactococcus lactis 1.8 após 24 horas.

Por fim, Tang, et al. (20201) demonstrou o aumento da concentração de todos os ácidos graxos de cadeia curta (fórmico, acético, propiônico e butírico), após $48 \mathrm{~h}$ de fermentação das frações de exopolissacarídeo obtidos de L. delbruecckii ssp. bulgaricus SRFM-1.

\subsection{Degradação do exopolissacarídeo na simulação do sistema gastrointestinal}

Tang, et al. (2020) ${ }^{1} 2$ verificaram, em ambos estudos, que após a simulação in vitro da digestão no sistema gastrointestinal, 2 frações de EPS (r-eps 2 e r-eps3) sintetizadas por Lactobacillus delbrueckii ssp. bulgaricus SRFM-1 apresentaram redução de $13.4 \%$ e $10.6 \%$ das massas, respectivamente, ao mesmo tempo a fração r-eps1 apresentou insignificante variação, esta foi a que obteve melhores taxas de fermentação por bactérias probióticas.

No estudo realizado por Grosu-Tudor, et al. (2013), todos os seis exopolissacarídeos selecionados ao passarem pela simulação do trato gastrointestinal superior não sofreram degradação, tanto os homopolissacarídeos, quanto os heteropolissacarídeo.

Foi observado por Hongpattarakere, et al. (2012) baixa percentagem de degradação do EPS W.cibaria A2 quando submetido a simulação gástrica intestinal. Já para Das, et al. (2014), os percentuais de digestão de exopolissacarídeo Lactobacillus plantarum DM5 foram irrelevantes.

\section{Discussão}

Apesar do acelerado crescimento de conhecimentos e da literatura científica sobre microbiota intestinal e prebióticos, esta revisão sistemática mostrou que as informações são muito limitadas no que se refere ao potencial do EPS de agir como um prebiótico. Não foi encontrada nenhuma revisão sistemática sobre esse tema.

Nos trabalhos analisados não há uma homogeneidade nos microrganismos utilizados para análise da produção de EPS, assim, torna-se difícil comparar os resultados e as diferenças na microbiota intestinal em cada estudo analisado.

Foi observado que os homopolissacarídeos (HOPS) apresentaram melhores resultados no estímulo do crescimento de bactérias não patogênicas Bifidobacterium bifidum, exceto os produzidos por Lactobacillus delbrueckii ssp. bulgaricus, que não apresentaram desenvolvimento de Bifidobacterium bifidum nos seus resultados.

Em todos os estudos, não houve degradação significativa dos EPSs homopolissacarídeos e heteropolissacarídeos que foram submetidos à simulação gastrointestinal. Isso se deve ao fato desses EPS possuírem efeito de fibra, ou seja, nenhum deles foi degradado durante a simulação pela passagem no sistema gastrointestinal, e com isso, eles podem atingir cólon sem serem hidrolisados ou absorvidos na parte superior do trato gastrointestinal (Grosu-Tudor, et al., 2013).

É importante a avaliação da produção de ácidos graxos de cadeia curta (AGCC) oriundos da fermentação de prebióticos, pois é um fator essencial para qualificar o potencial destes suplementos, visto que eles auxiliam na saúde do hospedeiro, como na criação de uma barreira para substâncias nocivas, assim como na redução de inflamação e de risco de câncer no intestino (Gibson, et al., 2017).

Entretanto, apesar dos estudos mostrarem que houve produção de ácido graxo, essa quantidade não foi especificada, e os trabalhos de Grosu-Tudor et al. (2013) e Hussein et al. (2015), não expõem se houve produção. Logo, não é possível chegar a uma conclusão em como de fato esse parâmetro interferiu nos estudos analisados.

As diferentes metodologias empregadas para a análise da ação prebiótica do EPS na microbiota intestinal dos ensaios estudados e as diferentes espécies de bactérias avaliadas inviabilizaram a determinação de um padrão específico que garanta a eficiência da ação prebiótica do EPS em sua totalidade. 
Outros aspectos que dificultam a comparabilidade dos resultados obtidos são a variabilidade das fontes de carbono utilizadas nos estudos. Porém, se observa que os trabalhos que usaram como fonte de carbono a sacarose, a produção de EPS foi muito significativa, então sugere-se que em futuros trabalhos a sacarose seja utilizada como única fonte de carbono.

Diante da heterogeneidade dos ensaios in vitro, evidencia-se a necessidade de padronização dos critérios e definições utilizados para permitir a comparação entre os microrganismos e sua produção de EPS, conforme mencionado na literatura. Portanto, independentemente das análises realizadas, não pode-se afirmar apenas com esses trabalhos que o EPS possui ação prebiótica em todos os casos.

\section{Conclusão}

A presente revisão sistemática constatou que EPS produzido por BAL apresentou influência no crescimento de Bifidobactérias assim como em cepas de bactérias ácido láticas probióticas, tais como as do gênero Lactobacillus. Tal influência não foi observada para o crescimento de bactérias não probióticas e patogênicas. Além disso, alguns do EPS testados possuem baixa degradação frente a passagem no sistema gastrointestinal. Com isso, o presente estudo sugere a incorporação de EPS em alimentos funcionais com o título de prebiótico, porém existe a necessidade de mais estudos para confirmar o tipo de EPS, duração do efeito e a dose de suplementação, a fim de aumentar as evidências dos resultados descritos pelos estudos.

\section{Referências}

Adesulu-Dahunsi, A. T., Jeyaram, K., Sanni, A. I., \& Banwo, K. (2018). Production of exopolysaccharide by strains of Lactobacillus plantarum YO175 and OF101 isolated from traditional fermented cereal beverage. PeerJ INC. 6, e5326. 10.7717/peerj.5326

Arora, K., Green, M., \& Prakash, S. (2020). The Microbiome and Alzheimer's Disease: Potential and Limitations of Prebiotic, Synbiotic, and Probiotic Formulations. Front. Bioeng. Biotechnol. 8:537847. 10.3389/fbioe.2020.537847

Caggianiello, G., Kleerebezem, M., \& Spano, G. (2016). Exopolysaccharides produced by lactic acid bacteria: from health-promoting benefits to stress tolerance mechanisms. Applied Microbiology and Biotechnology. 100(9), 3877-3886. 10.1007/s00253-016-7471-2

Daba, G. M., Elnahas, M. O., \& Elkhateeb, W. A. (2021). Contributions of exopolysaccharides from lactic acid bacteria as biotechnological tools in food, pharmaceutical, and medical applications. International Journal of Biological Macromolecules. 173, 79-89. 10.1016/j.ijbiomac.2021.01.110

Das, D., Baruah, R., \& Goyal, A. (2014). A food additive with prebiotic properties of an $\alpha$-d-glucan from Lactobacillus plantarum DM5. International Journal of Biological Macromolecules. 69, 20-26. 10.1016/j.ijbiomac.2014.05.029

Gao, Z., Fang, Y., Cao, Y., Liao, H., Nishinari, K., \& Phillips, G. O. (2017). Hydrocolloid-food component interactions. Food Hydrocolloids. 68, 149-156. doi 10.1016/j.foodhyd.2016.08.042

Gibson, G. R., Hutkins, R., Sanders, M. E., Prescott, S. L., Reimer, R. A., Salminen, S. J., Scott, K., Stanton, C., Swanson, K. S., Cani, P. D., Verbeke, K., \& Reid, G. (2017). Expert consensus document: The International Scientific Association for Probiotics and Prebiotics (ISAPP) consensus statement on the definition and scope of prebiotics. Nature Reviews Gastroenterology \& Hepatology. 14, 491-502. 10.1038/nrgastro.2017.75

Grosu-Tudor, S. S., Zamfir, M., Meulen, R. V. D., Falony, G., \& Vuyst, L. D. (2013). Prebiotic potential of some exopolysaccharides produced by lactic acid $\begin{array}{llll}\text { bacteria. Romanian Biotechnological Letters. } 18 & \text { (5), 8666-8676. } & \text { https://www.researchgate.net/publication/286068292_Prebio }\end{array}$ tic_potential_of_some_exopolysaccharides_produced_by_lactic_acid_bacteria

Guarner, F., Sanders, M. E., Eliakim, R., Fedorak, R., Gangl, A., Garisch, J., Kaufmann, P., Karakan, T., Khan, A. G., Kim, N., Paula, J. A. de., Ramakrihna, B., Shanahan, F., Szajewska, H., Thomson, A., \& Mair, A. L. (2017). Probiotics and prebiotics. World gastroenterology organization. https://www.worldgastroenterology.org/UserFiles/file/guidelines/probiotics-and-prebiotics-english-2017.pdf

Hongpattarakere, T., Cherntong, N., Wichienchot, S., Kolida, S., \& Rastall, R. A. (2012). In vitro prebiotic evaluation of exopolysaccharides produced by marine isolated lactic acid bacteria. Carbohydrate Polymers, 87(1), 846-852. 10.1016/j.carbpol.2011.08.085

Hussein, M. M., Ghaly, M. F., OSMAN, M. Y., Shalaby, A. S. G., \& Helal, M. M. I. (2015). Production and prebiotic activity of exopolysaccharides derived from some probiotics. Egyptian Pharmaceutical Journal. 14(1), 1. 10.4103/1687-4315.154687

Jindal, N. \& Khattar, S. J. (2018). Microbial Polysaccharides in Food Industry. Biopolymers for Food Design, 95-123. 10.1016 / b978-0-12-811449-0.00004-9

Liu, C., Kolida, S., Charalampopoulos, D., \& Rastall, R. A. (2020). An evaluation of the prebiotic potential of microbial levans from Erwinia sp. 10119. Journal of Functional Foods. 64, 103668. 10.1016/j.jff.2019.103668

Lybch, K. M, Coffey, A., \& Arendt, E. K. (2018). Exopolysaccharide producing lactic acid bacteria: Their technofunctional role and potential application in gluten-free bread products. Food Research International. 110, 52-61. 10.1016/j.foodres.2017.03.012 
Research, Society and Development, v. 10, n. 15, e194101522547, 2021

(CC BY 4.0) | ISSN 2525-3409 | DOI: http://dx.doi.org/10.33448/rsd-v10i15.22547

Maldonado-Contreras, A., Noel, S. E, Ward, D. V, Velez, M., \& Mangano, K. M. (2020). Associations between Diet, the Gut Microbiome, and Short-Chain Fatty Acid Production among Older Caribbean Latino Adults. Journal of the academy of nutrition and dietetics. 112(120), 2047-2060. 10.1016/ j.jand.2020.04.018

Moher, D., Liberati, A., Tetzlaff, J., \& Altman, D. G. (2009). Preferred reporting items for systematic reviews and meta-analyses: the PRISMA statement. PLoS medicine. 62 (10), 1006-1012. 10.1016/ j.jclinepi.2009.06.005

Renschler, M. A., Wyatt, A., Anene, N., Robinson-Hill, R., Pickerill, E. S., Fox, N. E., Griffith, J. A., \& McKillip, J. L. (2020). Using nitrous acid-modified de Man, Rogosa, and Sharpe medium to selectively isolate and culture lactic acid bacteria from dairy foods. Journal of Dairy Science. 103(2). 10.3168/jds.201917041

Tang, W., Zhou, J., Xu, Q., Dong, M., Fan, X., Rui, X., Zhang, Q., Chen, X., Jiang, M., Wu, J., \& Li, W. (20201). In vitro digestion and fermentation of released exopolysaccharides (r-EPS) from Lactobacillus delbrueckii ssp. bulgaricus SRFM-1. Carbohydrate Polymers. 230, 115593. 10.1016/j.carbpol.2019.115593

Tang, W., Han, S., Zhou, J., Xu, Q., Dong, M., Fan, X., Rui, X., Zhang, Q., Chen, X., Jiang, M., Wu, J., \& Li, W. (2020²). Selective fermentation of Lactobacillus delbrueckii ssp. Bulgaricus SRFM-1 derived exopolysaccharide by Lactobacillus and Streptococcus strains revealed prebiotic properties. Journal of Functional Foods. 69, 103952. 10.1016/j.jff.2020.103952

Zhou, Q., Feng, F., Yang, Y., Zhao, F., Du, R., Zhou, Z., \& Han, Y. (2018). Characterization of a dextran produced by Leuconostoc pseudomesenteroides XG5 from homemade wine. International Journal of Biological Macromolecules, 107, 2234-2241. 10.1016/j.ijbiomac.2017.10.098 